\title{
An improvement of a piecewise \\ curvature-corrected CMOS bandgap reference
}

\author{
Ruhaifi Abdullah Zawawi ${ }^{\mathrm{a}}$, Othman Sidek, \\ Wan Mohd Hafizi Wan Hassin, Mohamad Izat Amir Zulkipli, \\ and Nuha Rhaffor \\ Collaborative Microelectronic Design Excellence Centre (CEDEC), \\ Universiti Sains Malaysia (USM) \\ Engineering Campus, 14300, Nibong Tebal \\ Seberang Prai Selatan, Pulau Pinang, Malaysia \\ a) ruhaifi@cedec.usm.my
}

\begin{abstract}
In the current paper, an improvement of piecewise curvature-corrected CMOS bandgap reference (BGR) circuit is proposed. The circuit utilizes piecewise nonlinear curvature-corrected current (PNCCC) in a conventional BGR with a current control circuit, which compensates for the voltage reference at a higher temperature range. The current control circuit (CCC) is used to reduce the total current at low temperature when the PNCCC generator is inactive. The proposed circuit is realized in CMOS $0.13 \mu \mathrm{m}$ and has been verified to be able to save power consumption by $18.6 \%$ compared with a circuit without the current control circuit.
\end{abstract}

Keywords: bandgap reference circuit, piecewise curvature-corrected Classification: Integrated circuits

\section{References}

[1] K. Lasanen, V. Korkala, E. Raisanen-Ruotsalainen, and J. Kostamovaara, "Design of a 1 V Low Power CMOS Bandgap Reference Based on Resistive Subdivision," The 2002 45th Midwest Symposium on Circuits and Systems, 2002, vol. 3, pp. III-564-III-567, Aug. 2002.

[2] B. Razavi, Temperature Independent References, McGraw-Hill, City, 2001.

[3] G. C. M. Meijer, P. C. Schmale, and K. V. Zalinge, "A New CurvatureCorrected Bandgap Reference," IEEE J. Solid-State Circuits, vol. SC-17, no. 6, pp. 1139-1143, Dec. 1982.

[4] J. H. Li, X. B. Zhang, M. Y. Yu, and L. Han, "A 10 ppm 1.8-V Piecewise Curvature-Corrected Bandgap Reference in 0.5 $\mu \mathrm{m}$ CMOS," Asia Pacific Conference on Postgraduate Research in Microelectronics Electronics, 2009, pp. 416-419, Jan. 2009. 


\section{Introduction}

A reference circuit is an independent voltage or current source with a high degree of precision and stability. The output voltage or current should be independent of the power supply, temperature, and process variations [2]. Traditionally, the output voltage reference has always been approximately equal to the theoretical $1.22 \mathrm{eV}$ bandgap of silicon at $0 \mathrm{~K}$ [1].

The bipolar junction transistor (BJT) can be utilized in the BGR design. The properties of base-emitter voltage $V_{B E}$ are applied to generate the BGR signal. As the second-order non linearity of the function $V_{B E}(T)$ is generally the main limitation of the accuracy of the BGR [3], the bandgap reference circuit proposed in [4] has been reported to successfully compensated the nonlinear voltage. However, the proposed PNCCC generator in [4] consumes power, although the circuit remains inactive at low temperature. To avoid this problem, the control current circuit is proposed to suppress the current in the PNCCC generator without disturbing the output voltage variation.

\section{Proposed circuit}

The BGR circuit proposed by [4] utilizes a PNCCC generator to compensate for the nonlinear term of the first-order BGR in the higher temperature range only. The operation of the proposed circuit by [4] is illustrated in Fig. 1.

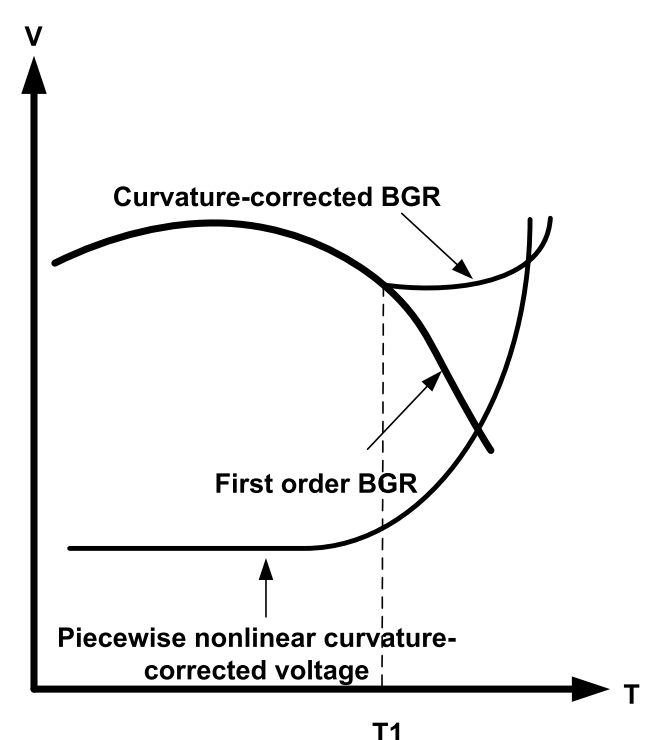

Fig. 1. Operation of the curvature-corrected BGR.

The temperature coefficient of the conventional BGR is slightly negative in the entire temperature range, resulting in small changes in the voltage reference for the temperature lower than $T_{1}$. A piecewise nonlinear curvaturecorrected voltage is added to the first-order BGR when the temperature is higher than $T_{1}$, significantly compensating for the nonlinear voltage in the first-order BGR circuit. The proposed circuit by [4] is shown in Fig. 2 (a).

The PTAT current $I_{P T A T}$, through the resistor $R_{4}$, produces a PTAT 


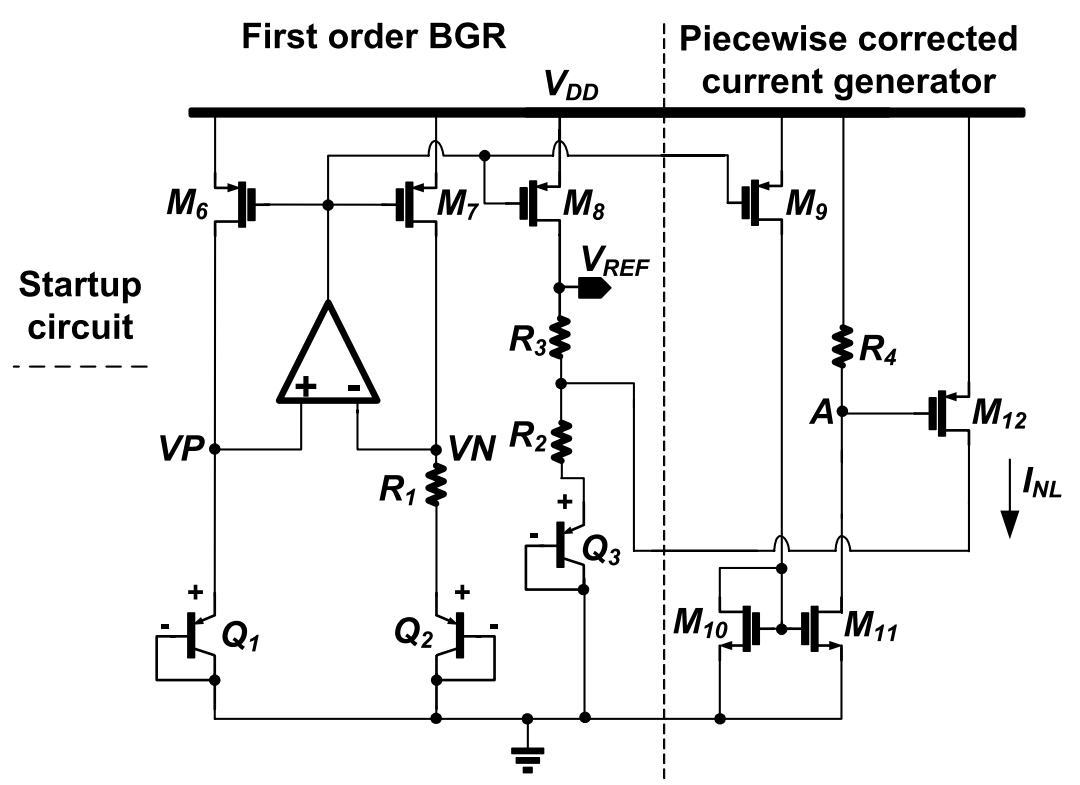

(a)

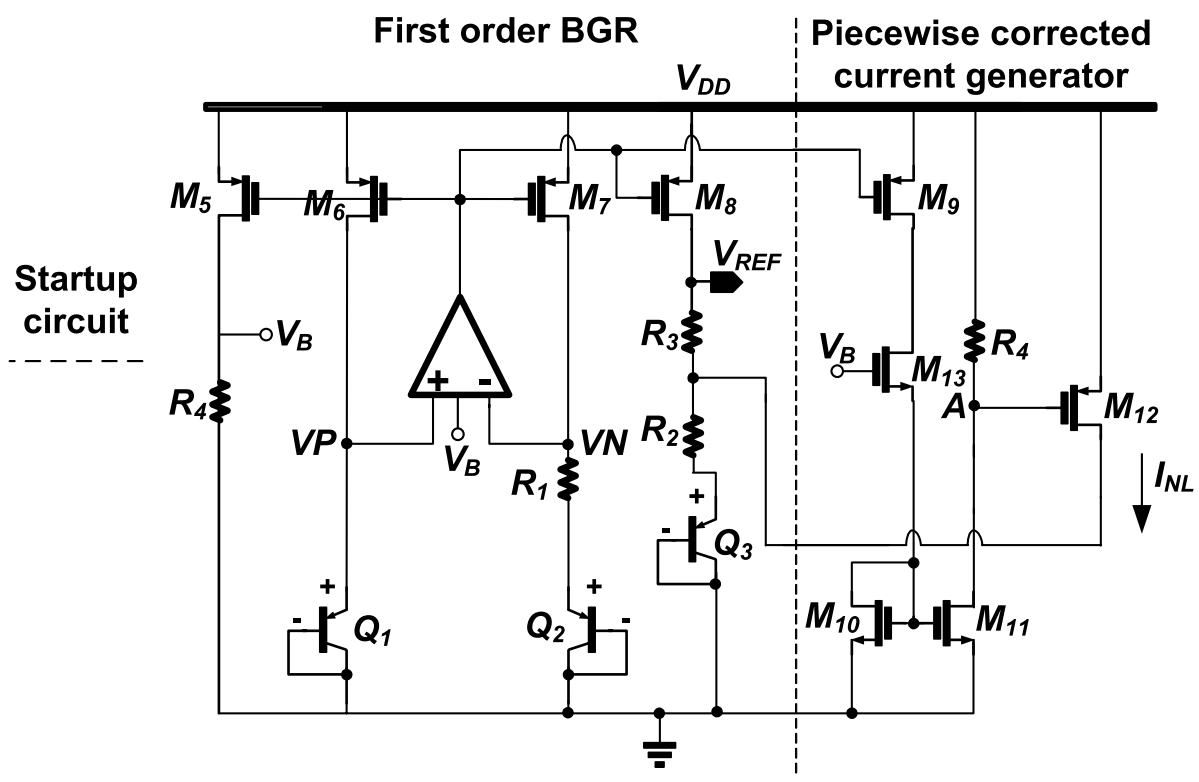

(b)

Fig. 2. (a) Previous BGR circuit proposed by [4], and (b) The proposed BGR circuit.

voltage given as

$$
V_{G_{M 12}}=R_{4} I_{P T A T}
$$

where

$$
I_{P T A T}=\frac{V_{T} \ln (n)}{R_{1}} .
$$

Parameter $n$ in Eq. (2) is the emitter-area ratio of $Q_{1}$ and $Q_{2}$. At a temperature below $T_{1}$, when the voltage $V_{S G_{M 12}}$ is much larger than its threshold voltage, $M_{12}$ operates in the saturation region. By increasing the temperature, $M_{12}$ will no longer be in the saturation region as it enters into the weak inversion region. When $V_{S G_{M 12}}$ is much lower than its threshold voltage, the transistor $M_{12}$ will be completely cut off, and no current will flow through 
$M_{12}$. The voltage reference $V_{R E F}$ of the curvature-corrected BGR can be derived from the circuit given as follows [4]:

$$
V_{R E F}=V_{E B Q 3}+\frac{V_{T} \ln (n)\left(R_{2}+R_{3}\right)}{R_{1}}+I_{N L} R_{2},
$$

where $I_{N L}$ is a nonlinear current flowing through $M_{12}$. Although Fig. 2 (a) works well in terms of output voltage compensation, the piecewise corrected current generator is not completely turned off for the temperature below $T_{1}$ because of the PTAT current flowing through transistor $M_{9}$.

We propose a BGR circuit along with a current control circuit in Fig. 2 (b). The transistor $M_{13}$ is added to the previous circuit and is biased with the PTAT voltage $V_{B} . V_{B}$ increases with temperature expressed as

$$
V_{B}=\frac{V_{T} \ln (n) R_{4}}{R_{1}}
$$

In the proposed circuit, $V_{B}$ is also used to bias the operational amplifier. The overall operation of the proposed BGR is similar to that of the circuit in Fig. 2 (a), except the current flowing through $M_{9}$, which is controlled by $M_{13}$. When the temperature is lower than $T_{1}$, the gate-source voltage of $M_{13}$ is less than its threshold voltage, resulting in the drain voltage of $M_{9}$ to be approximately equal to $V_{D D}$. A current near zero through $M_{9}$ occurs as $M_{9}$ enters the deep triode region. As the temperature increases, $M_{13}$ will be turned on when the gate-source voltage is larger than the threshold voltage. Conversely, the drain voltage of $M_{9}$ decreases with the increase in temperature, and the nonlinear current begin to flow through $M_{9}$ until it enters the saturation region. In this region, the PTAT current is generated, which is given as

$$
I_{P T A T}=\frac{V_{T} \ln (n)}{R_{1}}
$$

\section{Simulation}

We performed intensive simulations using an analog design environment and the Spectre-s simulator from Cadence on the proposed BGR circuit. The technology used was the $0.13 \mu \mathrm{m}$ CMOS with a $2.5 \mathrm{~V}$ supply voltage.

The nonlinear curvature-corrected current $I_{N L}$ and the current through $M_{9}$ are plotted in Fig. 3 (a). The minimum current through $M_{9}$ for the proposed circuit is only $12.9 \mathrm{nA}$ at $-50^{\circ} \mathrm{C}$, which differs by as much as $2.72 \mu \mathrm{A}$ from the BGR without the current control circuit. When the temperature reaches $40^{\circ} \mathrm{C}, M_{9}$ enters the saturation region and produces a linear current, as presented in the same figure. The current $I_{N L}$ is generated through $M_{12}$. The maximum current of $538 \mathrm{nA}$ is obtained at $125^{\circ} \mathrm{C}$.

Fig. 3 (b) shows the total current consumption and voltage reference within the temperature range of -50 to $125^{\circ} \mathrm{C}$. The minimum current of $29.3 \mu \mathrm{A}$ is achieved at $-50^{\circ} \mathrm{C}$ in the proposed BGR, whereas the previous BGR consumes $36.4 \mu \mathrm{A}$ in the same temperature. An improvement of $18.6 \%$ in terms of power consumption is achieved in the proposed BGR. The maximum and minimum output voltage references $V_{R E F}$ are 1.17984 and 


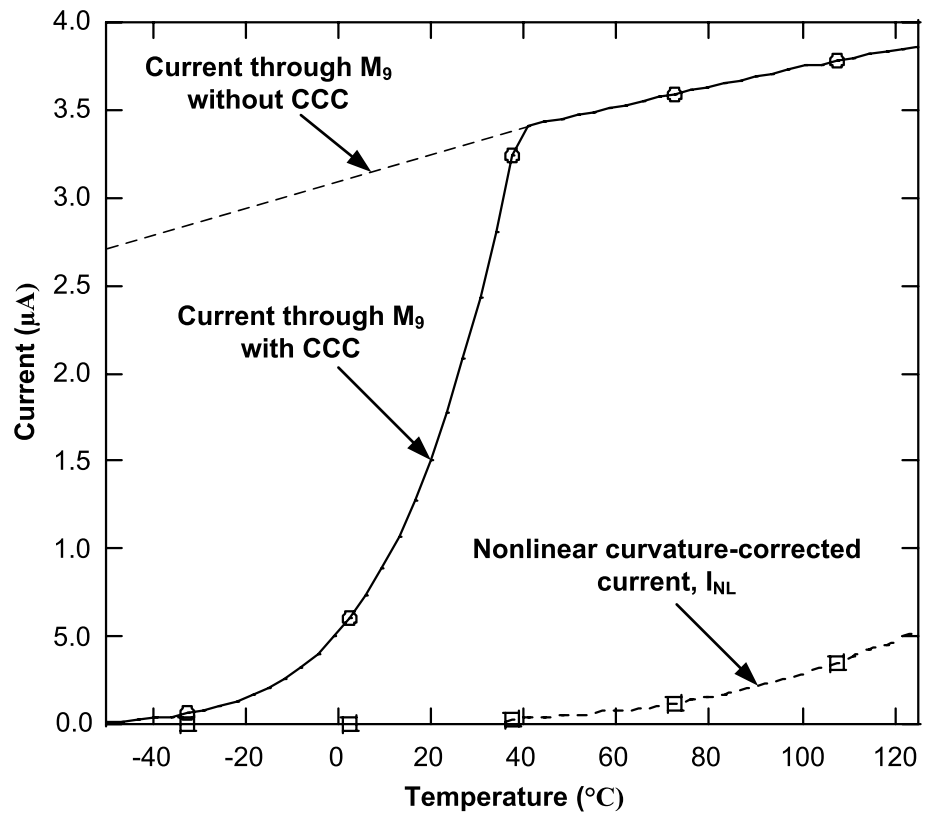

(a)

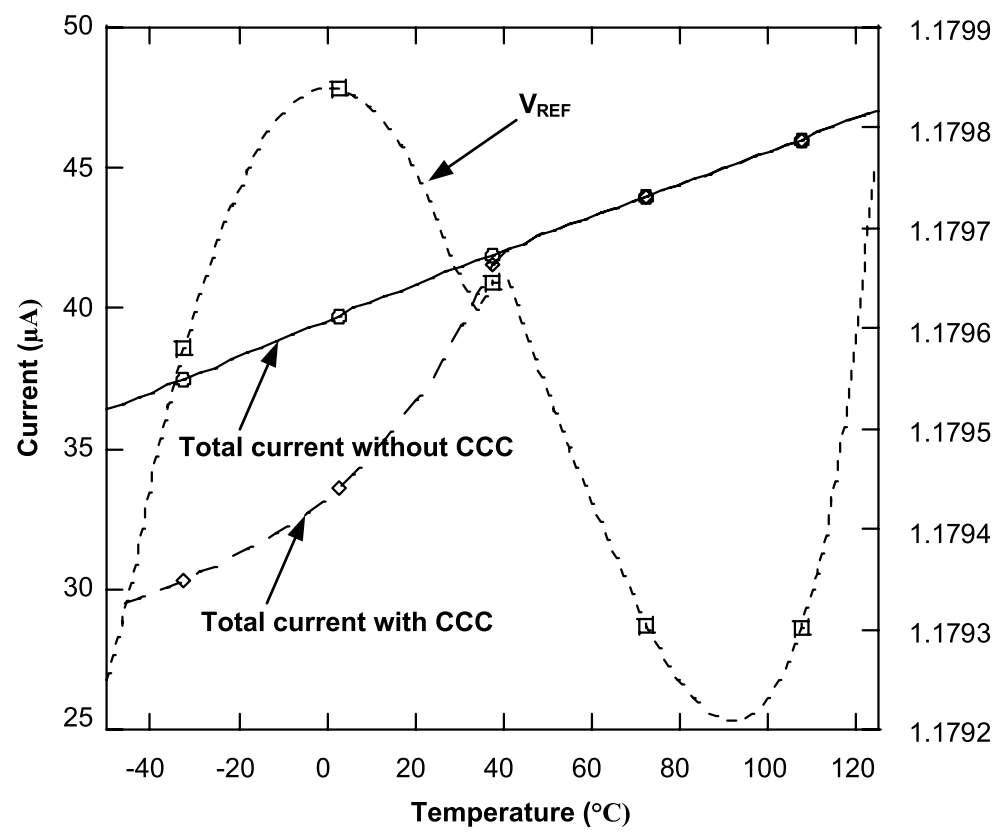

(b)

Fig. 3. (a) Current through $M_{9}$ and nonlinear curvaturecorrected current, and (b) Voltage reference and total current.

$1.17921 \mathrm{~V}$, respectively. Based on these values, the temperature coefficient is only $3.1 \mathrm{ppm} /{ }^{\circ} \mathrm{C}$.

\section{Conclusion}

An improvement of the piecewise curvature-corrected BGR circuit is presented. The minimum current flowing through $M_{9}$ in the proposed circuit is only $12.9 \mathrm{nA}$ at $-50^{\circ} \mathrm{C}$, whereas a current of $2.73 \mu \mathrm{A}$ is obtained from the 
previous circuit in the same temperature. The newly proposed circuit has been verified to be able to save power consumption up to $18.6 \%$ compared with the circuit without a current control circuit. The temperature coefficient of the voltage reference is $3.1 \mathrm{ppm} /{ }^{\circ} \mathrm{C}$ in the proposed BGR.

\section{Acknowledgments}

The authors would like to express their sincerest appreciation to all the Integrated Circuit Design Community (ICDC) group members, Silterra Malaysia Sdn. Bhd., and the Collaborative Microelectronic Design Excellence Centre (CEDEC). 